\title{
Modelling of metallic particle binders for increased part density in binder jet printed components.
}

\author{
Joseph W. Roberts ${ }^{a}$, Christopher J. Sutcliffe ${ }^{a}$, Peter L. Green ${ }^{b}$ and Kate Black ${ }^{{ }^{*}}$ \\ a School of Engineering, University of Liverpool, Harrison Hughes Building, Liverpool L69 3GH, \\ United Kingdom \\ b Institute for Risk and Uncertainty, University of Liverpool, Chadwick Building, Peach Street, \\ Liverpool L69 7ZF, United Kingdom
}

\begin{abstract}
:
Binder jet printed components typically have low overall density in the green state and high shrinkage and deformation after heat treatment. It has previously been demonstrated that, by including nanoparticles of the same material in the binder, these properties can be improved as the nanoparticles can fill the interstices and pore throats between the bed particles. The beneficial effects from using these additive binder particles can be improved by maximising the binder particle size, enabling the space within the powder bed to be filled with a higher packing efficiency. The selection of maximum particle size for a binder requires detailed knowledge of the pores and pore throats between the powder bed particles. In this paper, a raindrop model is developed to determine the critical radius at which binder particles can pass between pores and penetrate the bed. The model is validated against helium pycnometry measurements and binder particle drop tests. It is found that the critical radius can be predicted, with acceptable accuracy, using a linear function of the mean and standard deviation of the particle radii. Percolation theory concepts have been employed in order to generalise the results for powder beds that have different mean particle sizes and size distributions. The results of this work can be employed to inform the selection of particle sizes required for binder formulations, to optimise density and reduce shrinkage in printed binder jet components.
\end{abstract}

\section{Keywords}

Binder Jetting; Additive Manufacturing; Simulation; Powder bed; Density; Shrinkage

\section{Introduction:}

Many Additive Manufacturing (AM) processes, such as Laser Powder Bed Fusion (L-PBF), Direct Metal Laser Sintering (DMLS), Electron Beam Melting (EBM) and binder jet printing, rely on binding/melting or sintering individual powder grains within a powder bed to form a 'solid' part 1,2. In each of these processes, the packing density of the powder bed prior to printing, sintering and/or melting plays a significant role in determining the physical properties of the final part (such as density and shrinkage) ${ }^{3,4}$. The maximum achievable density of close-packed monodisperse spheres is around $74 \%{ }^{5}$ whereas powders used in AM processes tend to show densities of between $50 \%$ and $65 \%{ }^{5-7}$. Packing density is partly governed by the shape of the particles and the particle size distribution. Powder beds with a greater range of particle sizes tend to show increased packing density ${ }^{8,9}$ and powders used in AM processes typically have normal, log-normal or multimodal size distributions ${ }^{8,10,11}$. Several recent binder-jet studies have shown that the addition of particles into a binder system (hereby defined as 'binder particles'), with a smaller mean size than that of the powder bed, can reduce shrinkage and increase part density, improve strength and reduce the need for further infiltration during post processing ${ }^{12,13}$. To maximise part density, the particles added to 
the binder should be as large as possible to minimise open spaces within the bed, while also being small enough to pass between layers and promote inter-layer adhesion. Selection of the ideal size of particles to add to the binder requires advanced knowledge of the pores and pore throats left between particles in the powder bed. To the best of our knowledge, this paper is the first to define and calculate a critical radius for the infiltration of particles into randomly loose-packed powder beds with realistic size distributions.

\subsection{Simulation approaches for powder bed based additive manufacturing}

There are many different approaches to simulating various aspects of powder-based AM processes, e.g. binding, melting and solidification. A review of such simulations was published by M. Markl and C. Körner ${ }^{14}$. Concerning the generation of the powder bed, simulation approaches can be classified into one of three groups ${ }^{15}$ :

1) raindrop and sequential models, in which powder particles are sequentially placed into a specified simulation space under the influence of gravity, and allowed to roll over previously placed particles until a steady state is reached ${ }^{16-18}$,

2) non-sequential models, in which a dense array of overlapping particles is generated and then allowed to move or shrink to reduce the degree of particle overlap ${ }^{19,20}$ and

3) discrete element methods where dynamic inter-particle forces are considered during the particle placement ${ }^{9,21,22}$.

In this paper, a Monte-Carlo raindrop model is used to assemble multiple powder beds with a variety of particle size distributions (Phase 1). All of the particle size distributions are centred around typical powder sizes used for AM powder bed processes, as shown in the following references: $3,7,23,24$. Following phase 1 , a second raindrop model is used to infiltrate these powder beds with smaller particles (Phase 2). Percolation theory principles are then used to determine an equation for the binder particle critical radius $\left(r_{c}\right)$, based on the mean and standard deviation of the particle sizes in each powder bed. The results are normalised to allow application to powder bed distributions beyond those simulated for this work. The resulting equation for the critical radius is validated against the results of further simulations, for non-Gaussian size distributions, obtained via Scanning Electron Microscopy (SEM) image analysis of two typical AM powders supplied by Carpenter Additive and experimentally using $\mathrm{Cu}$ binder particles above and below the critical radius of a Ti powder bed.

\subsection{The application of percolation theory to binder jet printing}

Percolation theory has been used in many different research fields to model abrupt phase transitions in different media ${ }^{25,26}$. In one of percolation theory's simplest forms, a $2 \mathrm{D}$ square lattice is created with nodes at each lattice site. The lattice is populated with filled or empty sites according to a selected site probability ${ }^{*}$, where a probability of 0 gives only empty sites, and a probability of 1 fills in all sites. By changing the site probability (and given a lattice of sufficient size) a critical probability, $p_{c}$, can be determined. For an infinitely large lattice, site probabilities above $p_{c}$ give at least one continuous path along filled sites between opposite edges of the lattice and probabilities below $p_{c}$ do not. To illustrate, Figure 1 shows two $50 \times 50$ site grids for a four connected ${ }^{\dagger}, 2 \mathrm{D}$, site percolation network, with filled site probabilities above and below $p_{c}$. Below $p_{c}$ (Figure $1, \mathrm{~A}$ ) the network is made up of many small, discrete clusters. Above $p_{c}$ (Figure $1, \mathrm{~B}$ ) the network is dominated

\footnotetext{
* Sites are filled through the use of a site probability; a random number between 0 and 1 is generated for each site. If this number is less than or equal to the site probability then the site is filled.

${ }^{\dagger}$ i.e. sites can be connected above, below and to either side, but not along diagonals.
} 
by a single, large spanning cluster (i.e. there is a continuous, unbroken path between $y=0$ and $y=$ 50 , and between $x=0$ and $x=50$ ) and a few, discrete smaller clusters. As $p_{c}$ increases beyond 0.60 , the size of the spanning cluster grows and the number of small clusters reduces. In the simple case of an infinite, four connected, $2 \mathrm{D}$ square lattice, $p_{c}=0.5972$ for site percolation ${ }^{27}$.
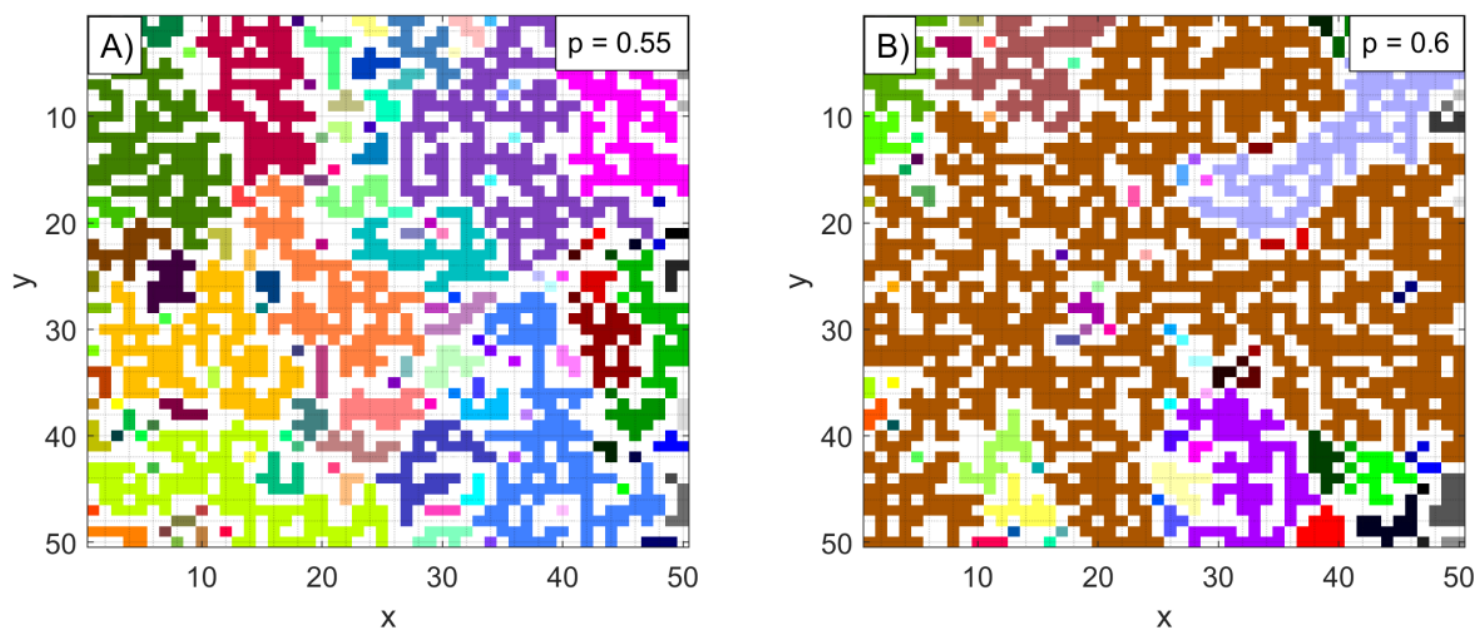

Figure 1: 2D, four connected, site percolation on a 50x50 site grid showing connected networks with the site probability $(A)$ below and $(B)$ above $p_{c}$. Each colour represents a single connected cluster and different coloured clusters have no connection between each other.

For the powder beds analysed in this paper, the percolation medium is defined as the spaces between powder particles, typically referred to as 'pores' for the larger open volumes and 'pore throats' for the constrictions running between them ${ }^{28}$. The 'percolate' is the small, added particles which would be delivered in the binder (see section 2.2).

If the binder particles have a sufficiently small radius $(r)$, then the probability of the particles passing through the full depth of the powder bed is unity and the entire pore space between particles will be open and fully connected. Conversely, if the binder particle is large enough, then the probability of the particles passing through the full depth of the powder bed will be zero; no particle will be able to pass the top surface of the powder bed or pass from any pore to another within the bed. Recalling that $r$ is the binder particle radius and $p_{c}$ is the critical probability for percolation, then, herein, $r_{p_{c}}$ is defined as the binder particle radius at the critical probability. As $r$ increases from $r_{p=1}$ to $r_{p=0}$ it stands that $p$ will decrease from 1 to 0 and that there will be a critical point for each powder bed distribution where $r=r_{p_{c}}$. At $r=r_{p_{c}}$, the limit of continuity between pores can be determined for a powder bed of a given depth where values of $r<r_{p_{c}}$ allow particles to move freely between pores and values of $r>r_{p_{c}}$ result in particles becoming stuck. Typically, percolation theory would be used to predict the critical values for an infinitely large network ${ }^{25,29,30}$. In the current work we are only concerned with network sizes beyond a few binder jet layer thicknesses $(30 \mu \mathrm{m}-100 \mu \mathrm{m}$, depending on the bed particle size ${ }^{7,8,24}$ ) as this is what is required to maximise part density and promote inter-layer adhesion during the printing process.

Work on pore structures between simulated, loose packed arrays of spherical particles has previously been carried out by Yang et al. ${ }^{22,28}$. This approach analysed arrays of single sized particles rather than distributions of particle sizes, and was focussed on the links between particle size, coordination number (the average number of contacts between particles), the shape of the open pores and the overall packing density. In contrast, the work presented here uses a variety of particle size distributions to better reflect real powder bed systems and effectively predicts the minimum 
radius of necks between bed particles, based on the mean and standard deviation of each powder bed.

\section{Simulation principles for the generation of powder beds and interaction of binder particles}

Powder bed generation (Phase 1) and the subsequent interactions of binder particles (Phase 2) were simulated using a Monte-Carlo geometric rain-drop model, similar to the model described by Zhou et al. ${ }^{15}$. Particles appear one by one at a height $100 \mu \mathrm{m}$ above the highest particle in the simulation space, at a random location in the $x y$ plane. If the particle is not in contact with any other particles in the simulation, its height is reduced by $10 \mathrm{~nm}$ each increment of the simulation. The distance to all other particles is determined at each increment and this process is repeated iteratively until the particle contacts another in the simulation. The Rodriguez rotation equation (equation 1$)^{31}$ is used to then rotate the moving particle $10 \mathrm{~nm}$ around the circumference of the contacted particle or particles:

$\boldsymbol{v}_{\text {rot }}=\boldsymbol{v} \cos (\theta)+(\boldsymbol{k} \times \boldsymbol{v}) \sin (\theta)+\boldsymbol{k}(\boldsymbol{k} \cdot \boldsymbol{v})(1-\cos (\theta))$

(where $\boldsymbol{v}_{\text {rot }}$ is the rotated vector, $\boldsymbol{v}$ is the original vector before rotation, $\boldsymbol{k}$ is the unit vector describing the axis of rotation, $\theta$ is the angle of rotation in radians and $\times$ and $\cdot$ are the cross product and dot product, respectively).

The axis of rotation is defined by the number of particles that the moving particle is in contact with. Particle motion is stopped once the moving particle reaches the base of the simulation or achieves a stable state, resting on previously placed particles. If the moving particle centre passes the centre of the contacted bed particle, the particle is detached and allowed to fall freely.

\subsection{Powder bed generation (Phase 1)}

In phase 1, to create a variety of powder beds, 17 sets of normally distributed particle radii were randomly generated through a program that generated Gaussian distributions based on a specified maximum and minimum particle size (Radii span, Table 1). A minimum of 5000 radii were generated for each distribution. For radii distributions with small mean particle sizes, which take up less volume per particle than those with larger mean radii, larger radii sets were generated (up to 15000 radii) to enable the $500 \mu \mathrm{m} \times 500 \mu \mathrm{m} \times 250 \mu \mathrm{m}$ simulation space to be filled. The bed mean particle radius $\left(\mu_{B}\right)$ and bed radius standard deviation $\left(\sigma_{B}\right)$ were calculated from the radii distributions and are used to identify and differentiate between the simulated powder beds. An example set with a fixed $\mu_{B}$ of around $18 \mu \mathrm{m}$ radius is shown in Figure $2(\mathrm{~A}) . \mu_{B}$ and $\sigma_{B}$ for all sets are shown in Table 1Error! Reference source not found..

Table 1: Gaussian distribution statistics of starting radii used for powder-bed generation.

\begin{tabular}{|c|c|c|c|}
\hline Radii set & Radii span $(\boldsymbol{\mu m})$ & Mean, $\mu_{B}(\boldsymbol{\mu m})$ & $\begin{array}{c}\text { Standard Deviation, } \\
\sigma_{B}(\boldsymbol{\mu m})\end{array}$ \\
\hline 1 & $4-26$ & 15.13 & 3.13 \\
\hline 2 & $6-26$ & 16.23 & 2.76 \\
\hline 3 & $8-26$ & 16.94 & 2.52 \\
\hline 4 & $10-26$ & 18.10 & 2.46 \\
\hline 5 & $12-26$ & 19.15 & 1.94 \\
\hline 6 & $14-26$ & 19.71 & 1.57 \\
\hline 7 & $16-26$ & 20.79 & 1.57 \\
\hline
\end{tabular}




\begin{tabular}{|c|c|c|c|}
\hline 8 & $4-32$ & 18.21 & 4.26 \\
\hline 9 & $6-30$ & 17.86 & 3.42 \\
\hline 10 & $8-28$ & 18.36 & 2.73 \\
\hline 11 & $10-26$ & 18.10 & 2.46 \\
\hline 12 & $12-24$ & 18.13 & 1.60 \\
\hline 13 & $14-22$ & 18.21 & 1.10 \\
\hline 14 & $16-20$ & 18.14 & 0.52 \\
\hline 15 & $4-20$ & 11.64 & 2.45 \\
\hline 16 & $10-26$ & 18.10 & 2.46 \\
\hline 17 & $16-32$ & 23.47 & 2.32 \\
\hline
\end{tabular}

25 powder beds were generated for each radii set, i.e. each $\mu_{B}$ and $\sigma_{B}$ combination. Steps were taken to ensure that the particle placements were random, without the near-close-packed placement that can occur when several particles of similar sizes rest upon a flat plane and begin to stack upon each other. In cases where several particles with similar sizes come to rest next to each other on a flat plane there is a high chance that they will adopt an hexagonally arranged close packed formation. This can then propagate through the simulation as more particles are added, leading to highly ordered domains with densities significantly higher than expected for random loose packing. To avoid flat walls at the simulation edges, no boundary conditions were set in the $x$ or $y$ planes during the bed generation. For each particle, an individual $z$ boundary was randomly selected between $z=0$ and $z=r_{B, \max }$, where $r_{B, \max }$ was the largest radius in the Gaussian particle distribution. The $z$ value was set this way in order to prevent a flat base to the simulation. After simulation, the pile of particles for each size distribution was cropped to include only particles with centres within a rectangular cuboid of size $x=500 \mu \mathrm{m}, y=500 \mu \mathrm{m}$ and $z=250 \mu \mathrm{m}$. For bed density calculations, a voxel map of the particles was generated and further cropped around the centre point to $x=350 \mu \mathrm{m}, y=350 \mu \mathrm{m}$ and $z=150 \mu \mathrm{m}$. The sizes were chosen to avoid artificially lowering the overall density through inclusion of the simulation edges. The powder bed generation process is illustrated for the $10 \mu \mathrm{m}-26 \mu \mathrm{m}$ radius distribution in Figure 2 . 

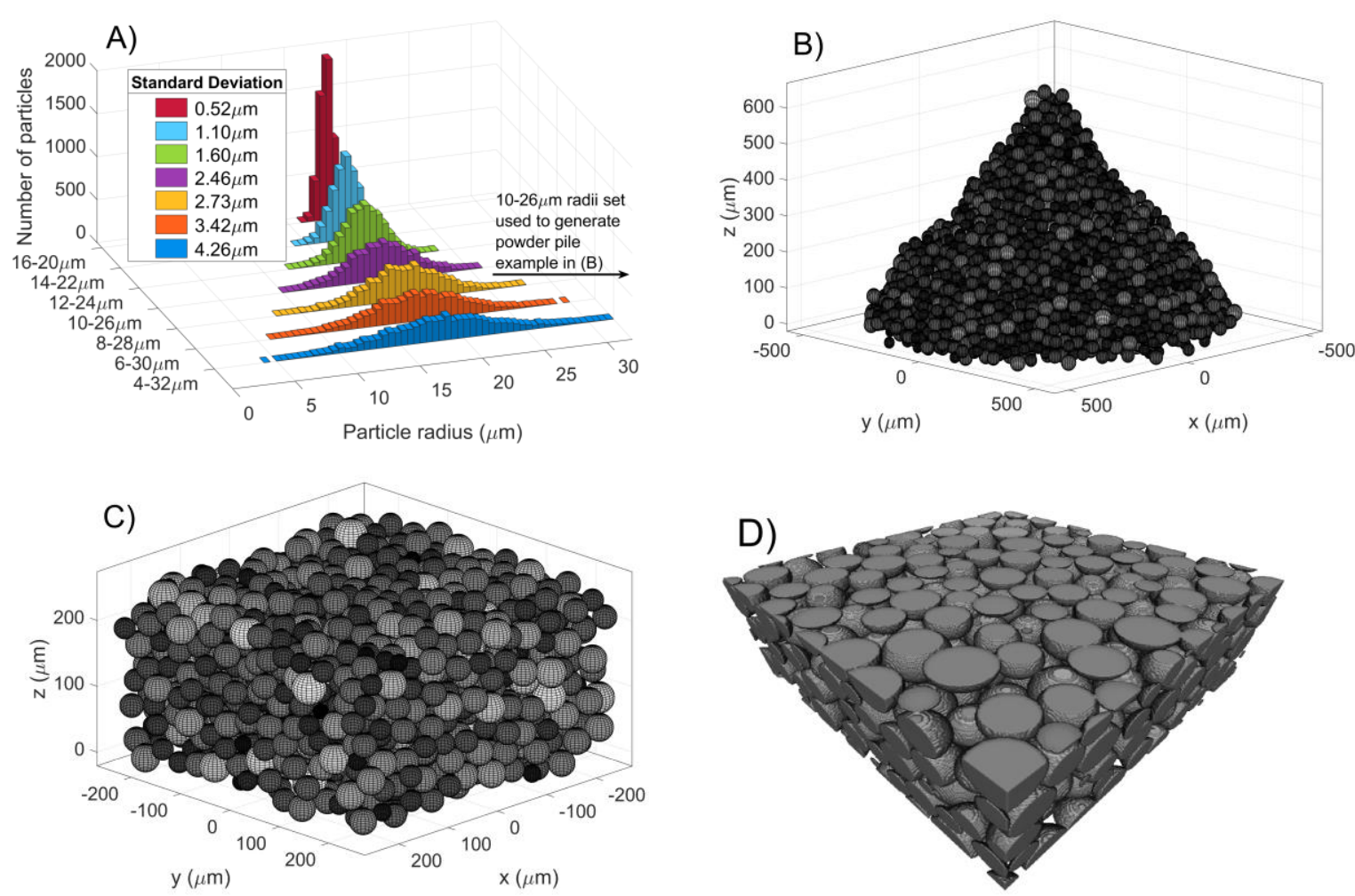

Figure 2: A) Histograms of normally distributed particle radii samples (sets 8 -14) used as the starting point for the simulation of powder beds. In the examples shown here, $\mu_{B}$ is around $18.1 \mu \mathrm{m}$ and $\sigma_{B}$ varies from $0.52 \mu \mathrm{m}$ to $4.26 \mu \mathrm{m}$. B) Simulated pile of 5000 particles using 10-26 $\mu \mathrm{m}$ radii set. C) Cropped particle pile including only particles with centres between $x$ and $y=-250 \mu \mathrm{m}$ to $250 \mu \mathrm{m}$ and $z=0 \mu \mathrm{m}$ to $250 \mu \mathrm{m}$ ( 1500 particles). D) Sliced particle bed (voxel mapped) used for density calculations (core volume shown, $350 \mu \mathrm{m}$ by $350 \mu \mathrm{m}$ by $150 \mu \mathrm{m}$ around centre point of cropped particle volume).

\subsection{Binder particle infiltration simulation (Phase 2)}

After the generation of the powder beds, a second set of simulations were run. Single particles, with radii ranging from $0.1 \mu \mathrm{m}$ to $10 \mu \mathrm{m}$ (increasing in $0.1 \mu \mathrm{m}$ steps), were dropped from random $x y$ locations above each powder bed and allowed to fall through. A circular drop area with a radius of $150 \mu \mathrm{m}$ over the centre of each powder bed was selected. The drop position and drop radius were chosen such that the binder particles had a low probability of escaping from the sides of the simulation before travelling the full depth of the bed, even if dropped at the edge of the drop radius. Once each binder particle had either fallen all the way through the simulation space or had reached a stable position within the bed, the start $z$ position and the final $z$ position were recorded along with the binder particle radius. The powder bed was reset to its original state after each infiltrated particle. This process was repeated 100 times for each infiltrated particle radius resulting in 10,000 binder particles dropped for each powder bed. As the starting radii were used to simulate 25 powder beds each, this gave a total of 250,000 binder particles dropped per bed radius size distribution and a total sample area of $\sim 1.75 \mathrm{~mm}^{2}$ per bed radius size distribution.

The probability of each binder particle of a given radius succeeding in traversing through the entire simulated powder bed was estimated by calculating the number of particles of a given radius that reached the base of the simulation, i.e. $z=0$, divided by the number of infiltration attempts. 


\subsection{Model Limitations}

\subsubsection{Model assumptions}

In both phase 1 and phase 2 the model uses geometric movement, collision and rolling rules to calculate the particle positions in each successive increment of the process. Other physical processes, such as the action of the liquid component of the binder, viscous, frictional and surface forces are not accounted for. It is also assumed that the powder bed particles remain locked in place once they reach a stable location, both during the addition of further bed particles and during the addition of binder particles. The model also assumes that bed and binder particles are perfect spheres.

\subsubsection{Model accuracy}

The model used to generate powder beds (phase 1) and to infiltrate binder particles into the powder beds (phase 2) moves particles in $10 \mathrm{~nm}$ increments. In phase 1 , as bed particles come into contact with each other, the rolling process (i.e. when the particle in motion begins to roll over one or more previously placed particles) begins once the distance between the centres of the particles is equal to or less than the sum of their radii. This typically results in a small overlap (between $0 \mathrm{~nm}$ and $10 \mathrm{~nm}$ ) between bed particles. However, the bed particles have a radii between $4 \mu \mathrm{m}$ and $32 \mu \mathrm{m}$, approximately 400 to 3200 times larger than the maximum overlap. Comparisons of regular, hexagonally packed particle arrays, with and without this overlap show a small difference in calculated density, with the overlapped arrays giving densities around $0.15 \%$ higher than the nonoverlapped arrays for simulation spaces with the same dimensions as used in the powder bed model. The particle overlap will also effect the results for the critical radius, in that particle edges would be marginally closer to each other, reducing the size of the gaps between them. However, we hypothesise that the effect is negligible, as the gaps are a few $10 \mathrm{~s}$ of $\mathrm{nm}$ smaller than without the particle overlap, below the resolution of the binder particle sizes tested $(100 \mathrm{~nm})$.

\section{Results and Discussion}

\subsection{Powder bed generation:}

25 loose-packed powder beds $(500 \times 500 \times 250 \mu \mathrm{m})$ were generated using the raindrop model for normally-distributed radii, as described in section 2.1. For validation, an additional 50 beds were generated using two sets of radii based on scanning electron microscope (SEM) image analyses of two different gas atomised metal powders, supplied by Carpenter Additive. The particle size distribution for the Carpenter Additive powders were determined following the algorithm developed by Atherton and Kerbyson ${ }^{32}$. To capture both the largest and smallest particles, multiple images at different magnifications were analysed. To adjust the results from each image so that they could be combined into a single histogram, the number of particles in each image were normalised with respect to the area of each image. Each particle radius histogram contains the results of around 10,000 particle sizes. Density calculations of the simulated powder beds were then compared to Helium pycnometry results obtained for these powders using a Quantachrome Instruments Multipycnometer (Table 2). In He pycnometry a sample chamber is filled with a known volume of the powder to be measured whilst a second reference chamber, with a known volume, is filled to a specified pressure (significantly above atmospheric pressure) with helium gas. The change in pressure of the total system as the reference chamber is emptied into the sample chamber allows the void fraction and the density of the powder to be calculated. Model bed densities were found to be within around $3 \%$ of those found by pycnometry, with the $20-53 \mu \mathrm{m}$ model underestimating the 
bed density by an average of around $1.5 \%$ and the $15-45 \mu \mathrm{m}$ model overestimating the bed density by an average of around $3 \%$. The model shows the correct trend in that the $20-53 \mu \mathrm{m}$ powder is denser than the $15-45 \mu \mathrm{m}$ powder. Some discrepancy is expected due to the assumptions used in the model; i.e. $100 \%$ spherical particles, no aggregation of particle clusters and zero frictional or mechanical forces between particles (see section 2.3 on model limitations). Simulated and measured powders showed densities consistent with the definition of randomly loose-packed spherical powders ${ }^{33}$, i.e. densities in the regions $55 \%$ to $60 \%$, compared to densities above $64 \%$ for random close-packing.

Table 2: Predicted powder bed densities and measured densities for Cu powders from LPW with 20-53 $\mu$ m and 15-45 4 m diameter distributions.

\begin{tabular}{|l|l|l|l|l|l|l|}
\hline Bed powder & $\begin{array}{l}\text { Average } \\
\text { density \% } \\
\text { (model) }\end{array}$ & $\begin{array}{l}\text { Maximum } \\
\text { density \% } \\
\text { (model) }\end{array}$ & $\begin{array}{l}\text { Minimum } \\
\text { density \% } \\
\text { (model) }\end{array}$ & $\begin{array}{l}\text { Standard } \\
\text { deviation } \\
\text { \% (model) }\end{array}$ & $\begin{array}{l}\text { Pycnometry } \\
\text { density \% } \\
\text { (experimental) }\end{array}$ & $\begin{array}{l}\text { Difference } \\
\text { (measured } \\
\text { - model) \% }\end{array}$ \\
\hline LPW 20-53 $\mu \mathrm{m}$ & 60.93 & 61.57 & 59.80 & 0.41 & 62.46 & +1.53 \\
\hline LPW 15-45 $\mu \mathrm{m}$ & 60.33 & 61.34 & 59.60 & 0.40 & 57.26 & -3.07 \\
\hline
\end{tabular}

\subsection{Percolation of binder particles and the binder particle critical radius}

In percolation theory, several definitions of the critical probability $\left(p_{c}\right)$ exist, depending on the concept of penetration in the percolation medium ${ }^{30}$. A common definition is the point (in terms of site probability) where the global structure changes from many, independent, small connected components to a single, large spanning component plus multiple, small, connected components ${ }^{34}$, i.e. the site probability at which a spanning cluster is likely to appear. For the case described in the current paper, the critical radius $\left(r_{c}\right)$ is defined as the largest binder particle radius where the probability of successfully traversing the powder bed is no longer unity. Figure 3 shows the normalised ${ }^{*}$ critical radius against the normalised powder bed standard deviation, measured from the simulated particle infiltration data. Radius values below $r_{C}$ have a bed traversal probability of 1 , and radius values above $r_{c}$ have a bed traversal probability of less than 1 . As the $\sigma_{B} / \mu_{B}$ ratio increases, the relative critical radius decreases. The linear model is shown in equation (2):

\footnotetext{
${ }^{*}$ Critical radius values and powder bed standard deviation values are normalised against the powder bed mean particle radius for each powder bed.
} 


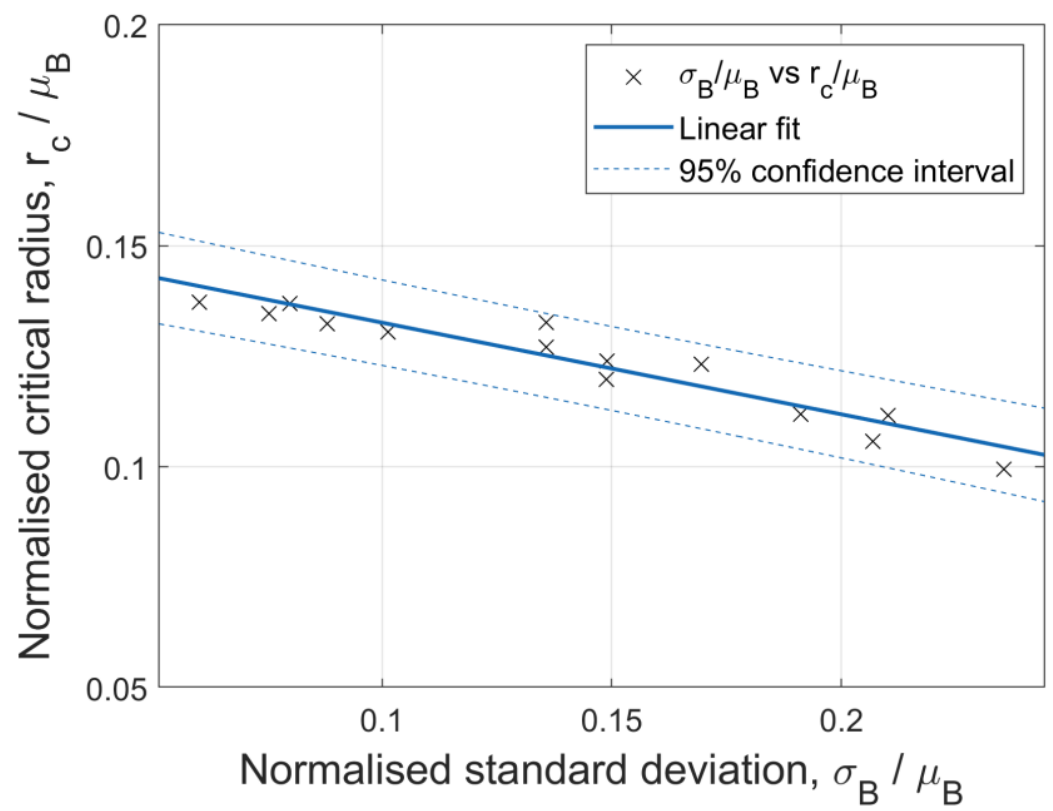

Figure 3: Linear regression of the normalised critical radii against normalised standard deviation for simulated powder beds.

$\overline{r_{c}}=c_{1}\left(\frac{\sigma_{B}}{\mu_{B}}\right)+c_{2}$

where $\bar{r}_{c}$ is the normalised critical radius, $c_{1}=-0.2074 \pm 0.05$ and $c_{2}=0.1534 \pm 0.007$. Figure 3 show the fit of this model, with an $R^{2}$ value of 0.881 . The dotted lines in Figure 3 represent a $95 \%$ confidence interval.

Rearranging equation (2), the critical radius is given by:

$r_{c}=c_{1} \sigma_{B}+c_{2} \mu_{B}$.

It should be noted that the critical radii presented here were all generated from simulated powder beds with densities ranging from $58 \%$ to $62 \%$. These densities are reasonable for loose-packed, spherical powders. However, real powders may undergo some densification in a binder jet system due to mechanical vibrations and compression from the powder delivery mechanism. This effect has been measured for spherical L-PBF powders spread using a rubber blade where it was found that, for powders with a tapped density of $\sim 65 \%$ and loose packed densities from $54 \%$ to $57 \%$, the single layer packing density was lower or similar to the loose packed density, ranging from $44 \%$ to $56 \%{ }^{7}$. We hypothesise that the relative densities of the powder will have little effect on the critical radius, effectively a measure of the width of the smallest pore throats present in the powder bed. The smallest possible pore throat within a powder bed should be the gap found between a close-packed cluster of three or four of the smallest particles within the distribution. As the particle sizes would not change as a powder densifies from random loose-packed through random close-packed to ordered close-packed, we would expect this minimum value to remain static. The critical radius is larger than the smallest possible neck for powder beds with depths less than infinity, as the probability of the cluster of smallest particles lying along a given percolation path is less than 1 . The smallest possible neck can be calculated by taking the values for the 3 smallest particles in the size distribution and applying Descartes' theorem ${ }^{35}$. In the beds tested in this paper, the finite powder bed depth of $250 \mu \mathrm{m}$ means that the probability of one of the smallest particle clusters being present is lower than one, and that binder particles may never encounter this minimum pore throat 
restriction. Another point of note is that the critical radius equation (equation 3 ) breaks down for powder beds were the standard deviation $\left(\sigma_{B}\right)$ approaches $73 \%$ of the value of the mean particle radius $\left(\mu_{B}\right)$. For powders beyond this relative standard deviation, equation (3) will yield a negative critical radius. Determining the precise $\sigma_{B} / \mu_{B}$ ratio at which the equation is no longer valid is currently a topic for future work. The $\sigma_{B} / \mu_{B}$ ratios simulated and tested in the current paper range from around zero to around $40 \%$.

\subsection{Application to real powder bed radii}

SEM image analysis (section 3.1) of two different gas atomised Cu powders was carried out to determine examples of real, non-Gaussian powder radii distributions (Figure 4) in order to validate the use of equation 3 when dealing with non-Gaussian distributions 25 powder beds for each of these non-Gaussian distributions were simulated using the histograms shown in Figure 4 (Phase 1) and subjected to the same particle infiltration process (Phase 2 ) as used for the previously discussed powder beds.
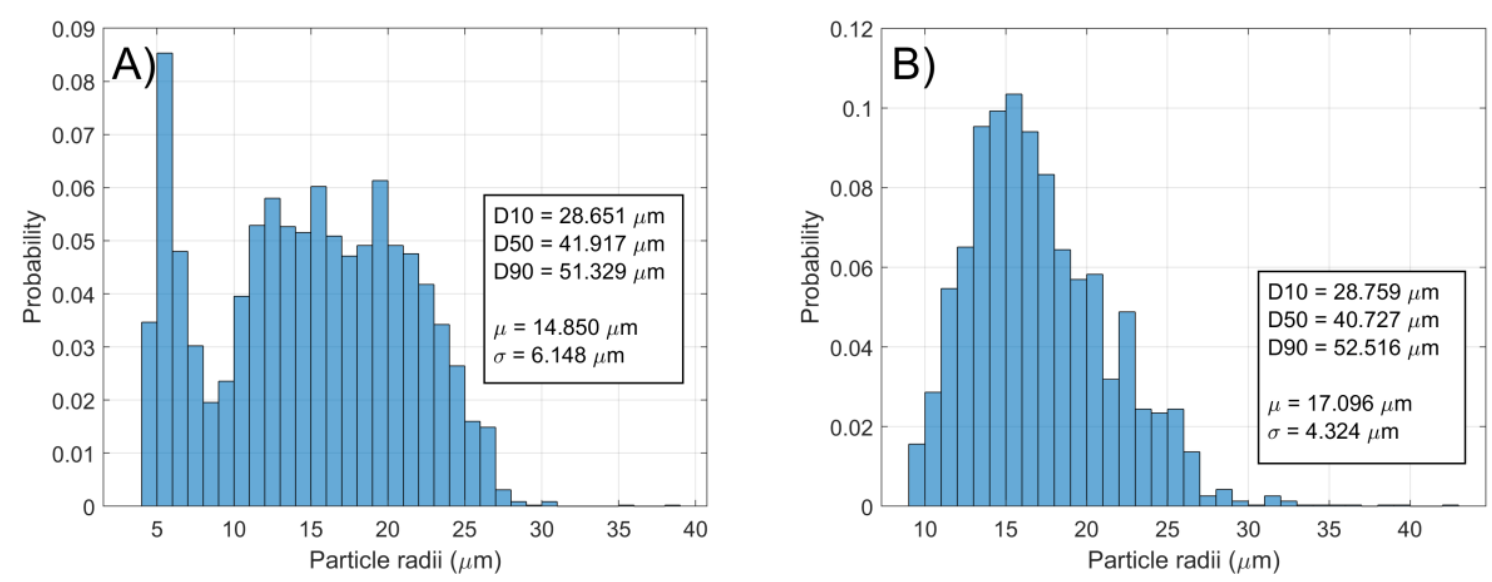

Figure 4: A) Histogram of LPW 20-53 $\mu$ m diameter powder from SEM image analysis. B) Histogram of LPW 15$45 \mu$ m diameter powder from SEM image analysis.

After the powder beds were simulated and cropped to the correct dimensions, the mean and standard deviation of the remaining particles $\left(\mu_{B}\right.$ and $\left.\sigma_{B}\right)$ were calculated for the remaining bed particles and found to be $14.96 \mu \mathrm{m}$ and $5.99 \mu \mathrm{m}$ for the $20-53 \mu \mathrm{m}$ powder and $16.94 \mu \mathrm{m}$ and $4.28 \mu \mathrm{m}$ for the $15-45 \mu \mathrm{m}$ powder. For the distributions shown in Figure 4 , it was found that there was sufficient statistical information in the first two moments of the distribution to allow the critical radius for each powder to be calculated using equation 3 . The critical radii determined by applying equation 3 to the radii distributions of particles that remained after the cropping process for both powders were $1.052 \mu \mathrm{m}$ and $1.711 \mu \mathrm{m}$. 

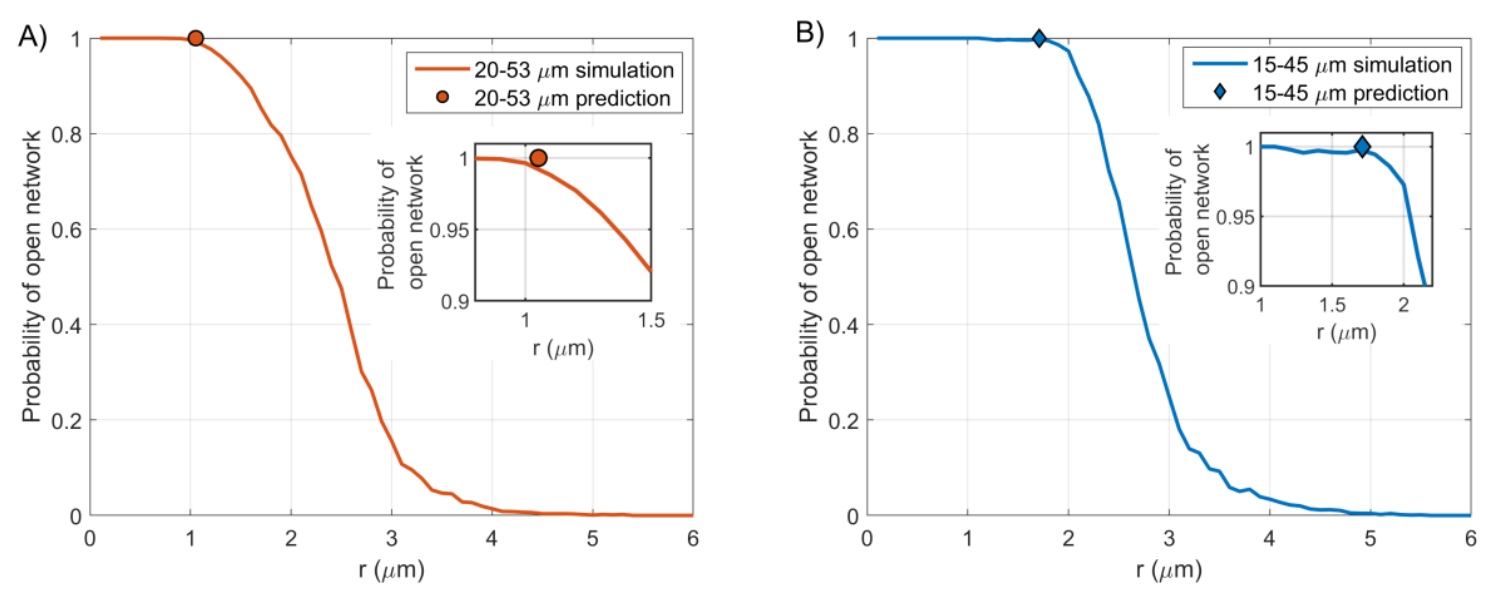

Figure 5: A) Critical radius prediction for 20-53 $\mu \mathrm{m}$ powder bed (Circle marker) and simulated open network probability (solid line). B) Critical radius prediction for 15-45 $\mu$ m powder bed (Diamond marker) and simulated open network probability (solid line). Insets in A and B show close-ups where the probability of an open network no longer equals 1 (solid lines) and the critical radius predicted by equation 3 (circle and diamond markers).

Figure 5 shows the simulated probability results from phase 2 and calculated critical radius predictions (i.e. calculated using equation 3 applied to the mean and standard deviation of the powder bed radii distributions) for binder particle infiltration into simulated powder beds based on non-Gaussian particle size distributions. It should be noted that the critical radius predictions (diamond and circle point markers, Figure 5 A and B, respectively) were calculated solely from the linear regression relationship inferred from the original simulations of normally distribution powder beds (i.e. equation 3), before implementing the model for phase 2 on the non-Gaussian powder beds. The simulation for phase 2 is represented by the continuous lines in Figure $5 \mathrm{~A}$ and $\mathrm{B}$. In both cases, the predictions made from equation 3 match closely with the onset of closed networks (i.e. $p$ $<1)$. For the 20-53 $\mu \mathrm{m}$ powder beds, the critical radius calculated from equation 3 is $1.052 \mu \mathrm{m}$ and the critical radius from the phase 2 simulations is between $0.9 \mu \mathrm{m}$ and $1.0 \mu \mathrm{m}$. For the $15-45 \mu \mathrm{m}$ powder beds, the critical radius calculated from equation 3 is $1.711 \mu \mathrm{m}$ and the critical radius from the phase 2 simulations is between $1.1 \mu \mathrm{m}$ and $1.8 \mu \mathrm{m}$. From the shape of the curve in Figure $5(\mathrm{~B})$, it appears that the critical radius is towards the higher end of the stated range. The lower value of 1.1 $\mu \mathrm{m}$, and the subsequent $0.7 \mu \mathrm{m}$ spread in the simulation prediction, may be attributed to the larger mean radius for the 15-45 $\mu \mathrm{m}$ powder which would reduce the total number of particles in the simulation and increase the noise in the probability results. Further validation is required to confirm whether the predictions made using the critical radius equation (equation 3)apply to further types of powder bed size distribution. Futher work is also required on the development of a mechanistic or probabilistic argument to link the various equation constants with the powder bed size distribution properties.

\subsection{Experimental validation}

The critical radius equation (equation 3 ) was experimentally validated using a commercially pure (CP) Ti bed powder and $\mathrm{Cu}$ binder particles dispersed in a reactive $\mathrm{Cu}$ ink binder. SEM image analysis was used to determine the particle size distribution of the CP Ti powder (Figure 6). The particle size distribution mean and standard deviation were $\mu_{B}=15.987 \mu \mathrm{m}$ and $\sigma_{B}=4.702 \mu \mathrm{m}$. The critical radius for the $\mathrm{CP}$ Ti powder, calculated using equation 3 , was determined to be $1.477 \mu \mathrm{m}$. 

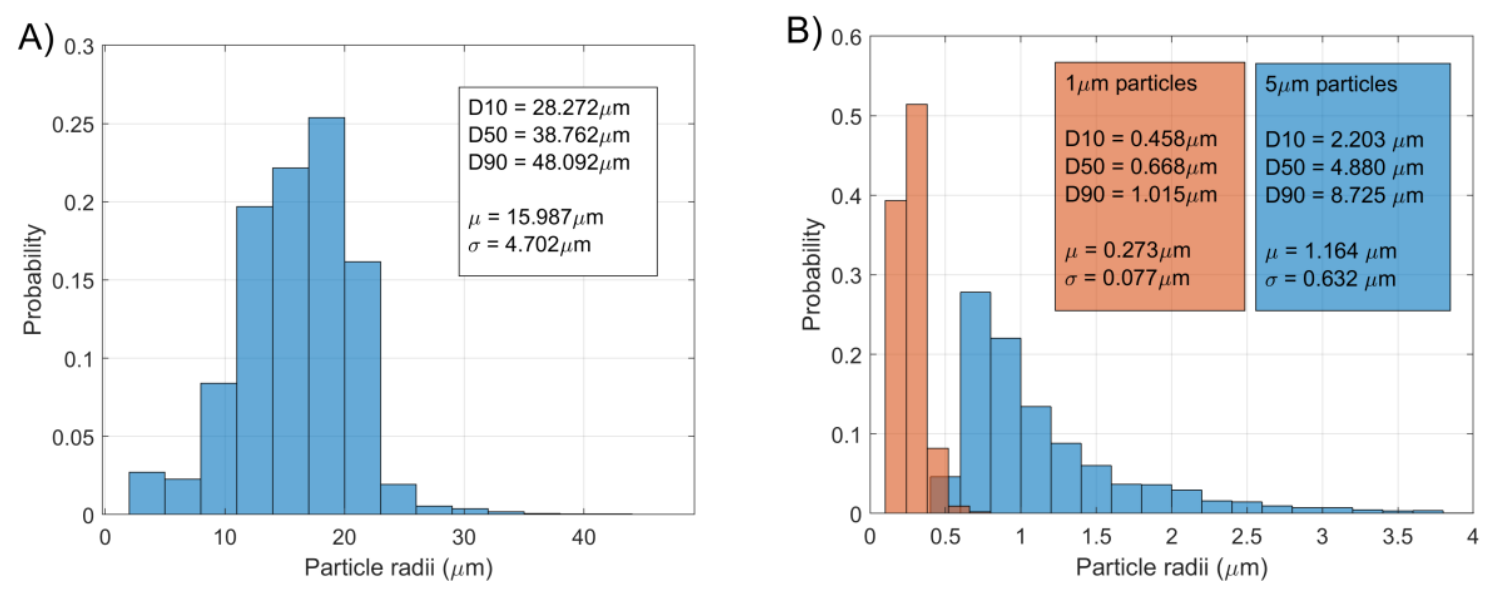

Figure 6: A) Particle radius size distribution for CP Ti powder. B) Particle radius size distributions for $1 \mu \mathrm{m}$ and 5 um diameter $\mathrm{Cu}$ powders.

Two different Cu powders, $0.5 \mu \mathrm{m}$ radius and $2.5 \mu \mathrm{m}$ radius as specified by the manufacturer, were dispersed into a solution of IPA and $\mathrm{Cu}$ binder ink containing $\mathrm{Cu}$ formate tetrahydrate, n-octylamine and 2-amino-2-methyl-1-propanol (AMP). Each dispersion contained $1 \mathrm{~g}$ of $\mathrm{Cu}$ ink, $4 \mathrm{~g}$ of IPA and $2.5 \mathrm{~g}$ of Cu particles, giving a particle concentration of $33 \%$ by mass. $100 \mu \mathrm{l}$ of each dispersion was pipetted onto the CP Ti powder bed in two $50 \mu \mathrm{l}$ drops using a micropipette from a height of $5 \mathrm{~mm}$. The second drop was applied once the first drop had soaked into the CP Ti powder. In the case of the $2.5 \mu \mathrm{m}$ particle ink, this took around $30 \mathrm{~s}$ and for the $0.5 \mu \mathrm{m}$ particle ink, around $5 \mathrm{~s}$. The powder wells measured $7.5 \mathrm{~mm}$ by $7.5 \mathrm{~mm}$ by $3.5 \mathrm{~mm}$, and as such represent between 35 and 110 layers in a powder based additive manufacturing system.
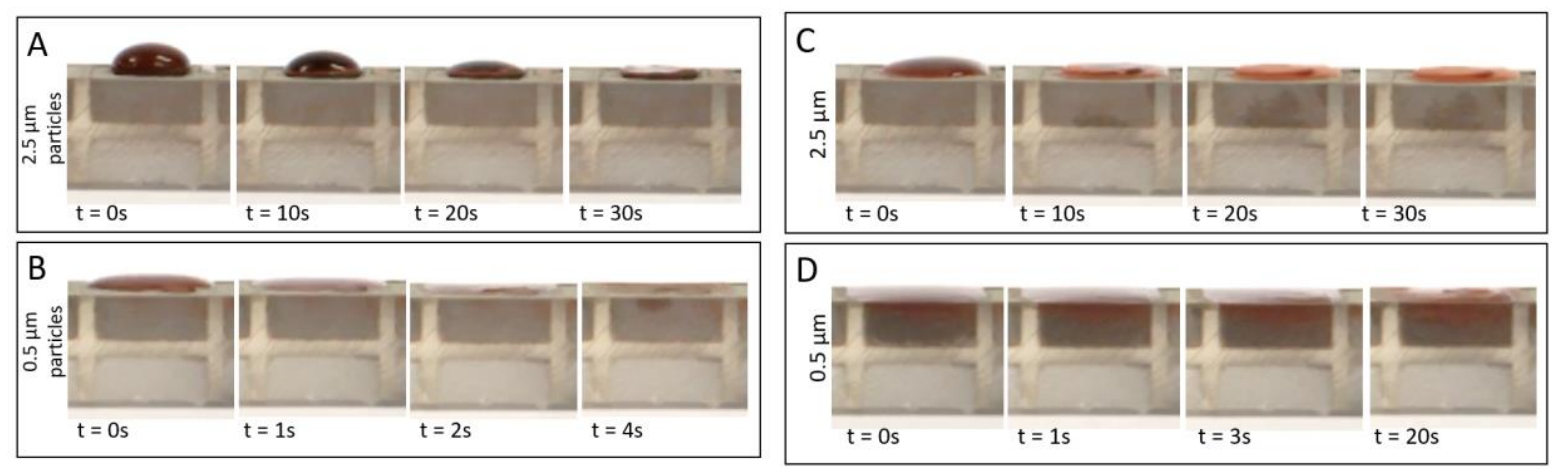

Figure 7: Drop tests for $2.5 \mu \mathrm{m}$ and $0.5 \mu \mathrm{m}$ Cu particle suspensions into CP Ti powder beds with $\mu_{B}=15.987 \mu \mathrm{m}$ and $\sigma_{B}=4.702 \mu \mathrm{m}$. A) $1^{\text {st }} 50 \mathrm{ml}$ drop with $2.5 \mu \mathrm{m}$ particles from $0 \mathrm{~s}$ to $30 \mathrm{s.} \mathrm{B)} 1^{\text {st }} 50 \mathrm{ml}$ drop with $0.5 \mu \mathrm{m}$ particles from $0 \mathrm{~s}$ to $4 \mathrm{~s}$. C) $2^{\text {nd }} 50 \mathrm{ml}$ drop with $2.5 \mu \mathrm{m}$ particles from $0 \mathrm{~s}$ to $30 \mathrm{~s}$. D) $2^{\text {nd }} 50 \mathrm{ml}$ drop with $0.5 \mu \mathrm{m}$ particles from $0 \mathrm{~s}$ to $20 \mathrm{~s}$.

According to the simulation results presented in section 3.2, binder particles with radii larger than the critical radius should not be able to penetrate a powder bed beyond 2.5 to 8 layers, depending on the layer thickness. Binder particles with radii smaller than the critical radius should be able to move freely past the upper layers of the bed. Figure 7 shows stills from video taken of the Cu particle binder drop tests into small wells containing CP Ti powder. For the Cu particles sizes above the CP Ti critical radius $\left(r_{C(T i)}=1.477 \mu \mathrm{m}\right), 2.5 \mu \mathrm{m}$ according to the manufacturer's specification and $\mu=1.164$ $\mu \mathrm{m}$ and $\sigma=0.632 \mu \mathrm{m}$ from SEM image analysis (Figure $6(\mathrm{~B})$ ), it can be seen in Figure 7(A) that the droplet rests on top of the Ti powder with a contact angle around $90^{\circ}$, indicating poor wetting of the Ti powder. Over the next $30 \mathrm{~s}$ after the drop impact, the liquid component of the drop, i.e. the $\mathrm{Cu}$ 
binder, IPA and stabiliser, penetrate into the Ti powder leaving a 'cake' of Cu particles at the surface and showing no visible evidence of the Cu particles being able to pass the upper layers of the $\mathrm{Ti}$ powder bed.

In Figure 7 (B), the Cu particles contained within the drop are smaller than the critical radius, specified as $0.5 \mu \mathrm{m}$ by the manufacturer and $\mu=0.273 \mu \mathrm{m}$ and $\sigma=0.077 \mu \mathrm{m}$ from SEM image analysis. The behaviour of the drop is markedly different from that of the drop with larger particle radii. At 0 s, the drop shows a much lower contact angle, around $30^{\circ}$, and spreads to cover a larger area of the Ti powder. Over the next $4 \mathrm{~s}$ both the liquid and solid components of the drop penetrate into the Ti powder bed leaving little disturbance at the surface. Through the translucent walls of the Ti powder well, it can be seen that the Cu powder has reached below the powder bed surface in some areas to a depth of around $1 \mathrm{~mm}$.

In Figure 7 (C) the second $50 \mathrm{ml}$ drop containing $2.5 \mu \mathrm{m}$ particles is pipetted onto the same area as the first drop. As with the first drop, it takes around 30s for the liquid component to penetrate the $\mathrm{Ti}$ powder and the Cu particles remain at the Ti powder surface. The liquid component can be seen penetrating portions of the Ti powder up to the full depth of the well. No colouration due to the presence of Cu particles is evident below the Ti powder surface.

Figure 7(D) shows the second drop containing $0.5 \mu \mathrm{m}$ Cu particles. As with the second drop containing the larger particles, the initial contact angle is reduced as the $50 \mathrm{ml}$ drop spreads to cover a larger area than the first. The liquid component of the drop quickly penetrates into the Ti powder, which shows almost complete wetting through the well side walls in less than $1 \mathrm{~s}$. A significant fraction of the Cu particles are also able to penetrate the Ti powder as shown by the change to an orange/pink colour in the top $1 \mathrm{~mm}$ of the Ti powder. The penetration depth of the $\mathrm{Cu}$ particles increases for the next $2 \mathrm{~s}$ and then slows, reaching a maximum depth of around $2 \mathrm{~mm}$, or 20 to 35 layers, at around $20 \mathrm{~s}$.

These results confirm the validity of the critical radius equation in predicting appropriate particle sizes to select when formulating particle containing binders for binder jet printing processes.

\subsection{Conclusions}

It has been shown that a raindrop model, using simple geometric rules, can replicate powder bed densities similar to those used in additive manufacturing processes. The powder bed model is validated against helium pycnometry density measurements. The critical radii necessary for binder particles to infiltrate the simulated powder beds (with Gaussian and non-Gaussian particle size distributions) have been determined via simulation, generalised and experimentally validated. The critical radius provides an upper limit on particle sizes to be used when optimising particulate binder formulations, enabling final part density to be maximised and minimise post-processing shrinkage.

\section{Acknowledgements}

This work has been supported by the Engineering and Physical Sciences Research Council (EPSRC EP/P027318/1).

\section{References:}

1. Liu, J., Jalalahmadi, B., Guo, Y. B., Sealy, M. P. \& Bolander, N. A review of computational modeling in powder-based additive manufacturing for metallic part qualification. Rapid Prototyping Journal (2018). doi:10.1108/RPJ-04-2017-0058 
2. Dini, F., Ghaffari, S. A., Jafar, J., Hamidreza, R. \& Marjan, S. A review of binder jet process parameters; powder, binder, printing and sintering condition. Met. Powder Rep. (2019). doi:https://doi.org/10.1016/j.mprp.2019.05.001

3. Rausch, A. M., Küng, V. E., Pobel, C., Markl, M. \& Körner, C. Predictive simulation of processwindows for powder bed fusion additive manufacturing: Influence of the powder bulk density. Materials (Basel). (2017). doi:10.3390/ma10101117

4. Rausch, A. M., Markl, M. \& Körner, C. Predictive simulation of process windows for powder bed fusion additive manufacturing: Influence of the powder size distribution. Computers and Mathematics with Applications (2018). doi:10.1016/j.camwa.2018.06.029

5. Scott, G. D. \& Kilgour, D. M. The density of random close packing of spheres. J. Phys. D. Appl. Phys. (1969). doi:10.1088/0022-3727/2/6/311

6. Stovall, T., de Larrard, F. \& Buil, M. Linear packing density model of grain mixtures. Powder Technol. (1986). doi:10.1016/0032-5910(86)80058-4

7. Wischeropp, T. M., Emmelmann, C., Brandt, M. \& Pateras, A. Measurement of actual powder layer height and packing density in a single layer in selective laser melting. Additive Manufacturing (2019). doi:10.1016/j.addma.2019.04.019

8. Muñiz-Lerma, J. A., Nommeots-Nomm, A., Waters, K. E. \& Brochu, M. A comprehensive approach to powder feedstock characterization for powder bed fusion additive manufacturing: A case study on AISi7Mg. Materials (Basel). (2018). doi:10.3390/ma11122386

9. Lee, Y. S., Nandwana, P. \& Zhang, W. Dynamic simulation of powder packing structure for powder bed additive manufacturing. Int. J. Adv. Manuf. Technol. (2018). doi:10.1007/s00170018-1697-3

10. Mostafaei, A., Rodriguez De Vecchis, P., Nettleship, I. \& Chmielus, M. Effect of powder size distribution on densification and microstructural evolution of binder-jet 3D-printed alloy 625. Mater. Des. (2019). doi:10.1016/j.matdes.2018.11.051

11. Heiden, M. J. et al. Evolution of $316 \mathrm{~L}$ stainless steel feedstock due to laser powder bed fusion process. Addit. Manuf. (2019). doi:10.1016/j.addma.2018.10.019

12. Bai, Y. \& Williams, C. B. The effect of inkjetted nanoparticles on metal part properties in binder jetting additive manufacturing. Nanotechnology (2018). doi:10.1088/1361$6528 /$ aadObb

13. Elliott, A., AlSalihi, S., Merriman, A. L. \& Basti, M. M. Infiltration of Nanoparticles into Porous Binder Jet Printed Parts. Am. J. Eng. Appl. Sci. (2016). doi:10.3844/ajeassp.2016.128.133

14. Markl, M. \& Körner, C. Multiscale Modeling of Powder Bed-Based Additive Manufacturing. Annu. Rev. Mater. Res. (2016). doi:10.1146/annurev-matsci-070115-032158

15. Zhou, J., Zhang, Y. \& Chen, J. K. Numerical Simulation of Random Packing of Spherical Particles for Powder-Based Additive Manufacturing. J. Manuf. Sci. Eng. (2009). doi:10.1115/1.3123324

16. Visscher, W. M. \& Bolsterli, M. Random packing of equal and unequal spheres in two and three dimensions. Nature (1972). doi:10.1038/239504a0

17. Körner, C., Attar, E. \& Heinl, P. Mesoscopic simulation of selective beam melting processes. J. Mater. Process. Technol. (2011). doi:10.1016/j.jmatprotec.2010.12.016

18. Shi, Y. \& Zhang, Y. Simulation of random packing of spherical particles with different size 
distributions. in Applied Physics A: Materials Science and Processing (2008). doi:10.1007/s00339-008-4547-6

19. Kansal, A. R., Torquato, S. \& Stillinger, F. H. Computer generation of dense polydisperse sphere packings. J. Chem. Phys. (2002). doi:10.1063/1.1511510

20. Farr, R. S. \& Groot, R. D. Close packing density of polydisperse hard spheres. J. Chem. Phys. (2009). doi:10.1063/1.3276799

21. Meier, C., Weissbach, R., Weinberg, J., Wall, W. A. \& John Hart, A. Modeling and characterization of cohesion in fine metal powders with a focus on additive manufacturing process simulations. Powder Technol. (2019). doi:10.1016/j.powtec.2018.11.072

22. Yang, R. Y., Zou, R. P. \& Yu, A. B. Computer simulation of the packing of fine particles. Phys. Rev. E - Stat. Physics, Plasmas, Fluids, Relat. Interdiscip. Top. (2000). doi:10.1103/PhysRevE.62.3900

23. Nan, W. \& Ghadiri, M. Numerical simulation of powder flow during spreading in additive manufacturing. Powder Technol. (2019). doi:10.1016/j.powtec.2018.10.056

24. Markl, M. \& Körner, C. Powder layer deposition algorithm for additive manufacturing simulations. Powder Technol. (2018). doi:10.1016/j.powtec.2018.02.026

25. Essam, J. W. Percolation theory. Reports on Progress in Physics (1980). doi:10.1088/00344885/43/7/001

26. Saberi, A. A. Recent advances in percolation theory and its applications. Physics Reports (2015). doi:10.1016/j.physrep.2015.03.003

27. Christensen, K. Percolation theory notes. Thesis (2002).

28. Yang, R. Y., Zou, R. P., Yu, A. B. \& Choi, S. K. Pore structure of the packing of fine particles. J. Colloid Interface Sci. (2006). doi:10.1016/j.jcis.2006.02.041

29. Powell, M. J. Site percolation in randomly packed spheres. Phys. Rev. B (1979). doi:10.1103/PhysRevB.20.4194

30. Wierman, J. C. On critical probabilities in percolation theory. J. Math. Phys. (2003). doi:10.1063/1.523894

31. Rodriguez, O. Des lois géometriques qui regissent les deplacements d'un système solide independament des causes qui peuvent les produire. J. Math. Pures Appl. (1840).

32. Atherton, T. J. \& Kerbyson, D. J. Size invariant circle detection. Image Vis. Comput. (1999). doi:10.1016/S0262-8856(98)00160-7

33. Kawakatsu, T., Nakajima, M., Nakao, S. ichi \& Kimura, S. Three-dimensional simulation of random packing and pore blocking phenomena during microfiltration. Desalination (1995). doi:10.1016/0011-9164(95)00023-U

34. Janson, S. \& Warnke, L. On the critical probability in percolation. Electron. J. Probab. (2018). doi:10.1214/17-EJP52

35. Lagarias, J. C., Mallows, C. L. \& Wilks, A. R. Beyond the descartes circle theorem. Am. Math. Mon. (2002). doi:10.2307/2695498 